\title{
Purpura Fulminans in an adult with Pseudomonas aeruginosa septicemia
}

\section{Leah Ellis Wells', Alexander Kennon²}

\author{
${ }^{1}$ Medical Student at Mercer University School of Medicine, ${ }^{2}$ Dermatologist at Skin Care Physicians of Georgia in Macon, GA \\ Corresponding author: Leah Ellis Wells, E-mail: Leah.C.Ellis@live.mercer.edu
}

\begin{abstract}
Purpura fulminans is a serious dermatological condition that can be a sequela of sepsis complicated by disseminated intravascular coagulopathy (DIC). This occurs most frequently in children with Neisseria meningitis or Streptococcus pneumoniae infection. Once diagnosed, treatment of the underlying infection, surgical debridement, and replacement of anticoagulation factors are recommended. Most patients suffer significant long-term morbidity, including amputations and sometimes death. We describe a rare case of an adult patient with Pseudomonal bacteremia leading to purpura fulminans. The patient's underlying infection was promptly treated and her lesions were debrided appropriately. However, correction of coagulopathy was not pursued. Despite this, amputations were avoided and the patient survived. This case demonstrates the need to be vigilant and open minded to the diagnosis of dermatological diseases with atypical presentations. Further, it highlights the chief importance of treating the underlying infection and providing proper wound care in septic purpura fulminans.
\end{abstract}

Key words: Purpura fulminans; Pseudomonas aeruginosa; Sepsis

\section{INTRODUCTION}

Purpura fulminans is a rapidly progressive thrombotic disorder characterized by hemorrhagic infarction of skin and disseminated intravascular coagulation. It is associated with high mortality and long-term morbidity in survivors, making recognition and treatment crucial. It most commonly occurs in infants and children in association with a heritable deficiency of protein $\mathrm{C}$ or protein $\mathrm{S}$ and/ or an acquired deficiency secondary to an infection with N. meningitis or S. pneumoniae [1]. Purpura fulminans in an adult with Pseudomonal sepsis is not typical [2-4].

Diagnosis is based on recognition of erythematous painful macules that develop central areas of necrosis as the disease progresses. Typical laboratory values include prolonged plasma clotting times, thrombocytopenia, and markedly reduced Protein $\mathrm{C}$ and Protein S levels [1]. Biopsy can be performed to confirm diagnosis, but empiric treatment should be initiated while waiting for the results [2]. Standard treatment of purpura fulminans includes antibiotic therapy for any underlying infection, surgical debridement to remove necrotic tissue, and correction of the various coagulation cascade abnormalities characteristic of the disease [1,5].

\section{CASE REPORT}

A female in her 60s was admitted to the intensive care unit for altered mental status. Upon arrival she was afebrile, tachycardic, tachypnic, and hypotensive, but able to follow simple commands. On physical examination, there was tenderness and erythema of the right lower extremity. Initial labs revealed a leukocytosis and lactic acidosis. An initial platelet count was $140\left(10^{3}\right) /$ uL. Blood cultures were obtained, and the patient was started on empiric broad-spectrum antibiotics and pressors. When the blood culture was positive for Pseudomonas aeruginosa, antibiotics were tailored to sensitivities accordingly. Despite therapy, the patient began developing stellate dark necrotic lesions with bullae (Figs. 1 - 3). At this time, D-dimer, prothrombin time (PT), and partial thromboplastin time (PTT)

\footnotetext{
How to cite this article: Wells LE, Kennon A. Purpura Fulminans in an adult with Pseudomonas aeruginosa septicemia. Our Dermatol Online. 2018;9(4):404-406.

Submission: 06.04.2018; Acceptance: 11.06.2018

DOI:10.7241/ourd.20184.10
} 


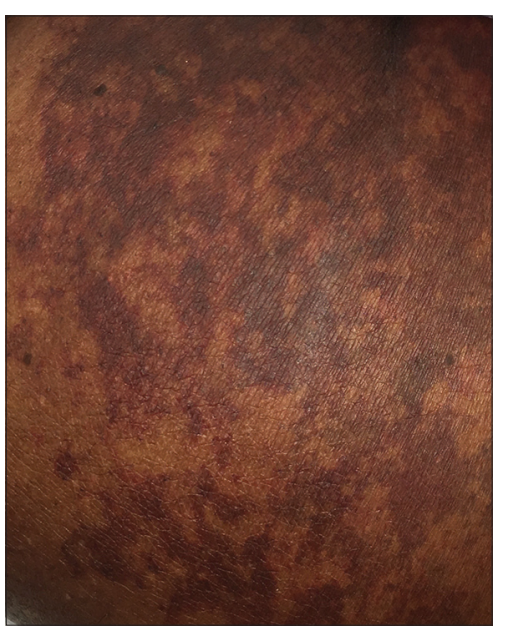

Figure 1: Violacious purpuric stellate patches on right lower extremity.

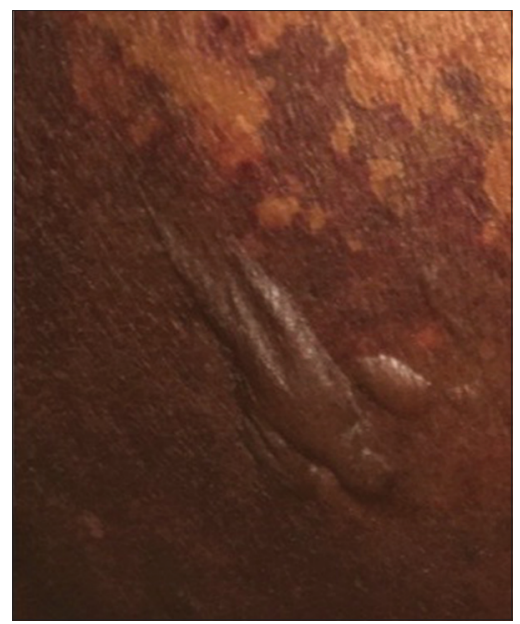

Figure 2: Large flaccid bullae within field of purpuric patches on right lower extremity.

were elevated and platelet count was $54\left(10^{3}\right) / \mathrm{uL}$. Given clinical presentation and laboratory findings, a diagnosis of purpura fulminans was made. Subsequent biopsy confirmed diagnosis. Appropriate antibiotics were continued and the patient underwent several surgical debridements. She did not receive heparin, fresh frozen plasma, protein $\mathrm{C}$ supplementation, antithrombin, or platelet transfusion. She was discharged to a rehab facility after a prolonged hospital stay.

\section{DISCUSSION}

Purpura fulminans is a rare, life threatening dermatological emergency with multiple etiologies, each typically seen in a certain population. Neonatal purpura fulminans is usually due to a heritable deficiency of Protein $\mathrm{C}$ or Protein S [1]. Post-infectious purpura fulminans may appear in children a few days to weeks after a febrile infectious illness. Affected children typically show a

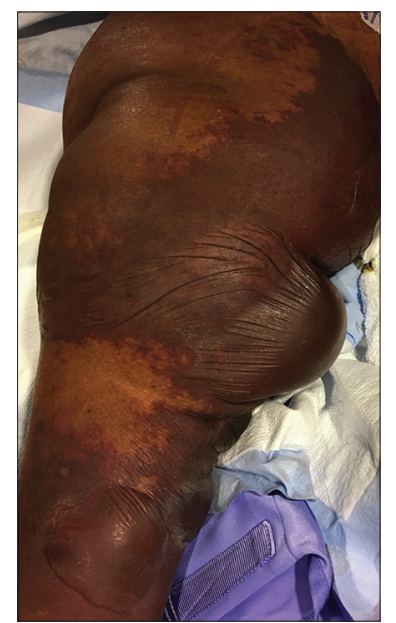

Figure 3: Right lower extremity displaying extensive involvement several days into antibiotic treatment, prior to surgical debridement.

severe acquired Protein S deficiency resulting from $\operatorname{Ig} \mathrm{G}$ autoantibodies that cross-react with Protein $S$, increasing its clearance and leading to a hypercoagulable state. Acute sepsis secondary to certain organisms, such as Neisseria meningitis, Streptococcus pneumonia, and Haemophilus influenzae can also precipitate purpura fulminans, most commonly in children [1]. The pathogenesis of acute infectious purpura fulminans is related to the systemic activation of coagulation and complement pathways, which commonly accompanies sepsis and DIC [2]. In adults, infectious PF is most commonly caused by sepsis from similar organisms as children. Purpura fulminans secondary to Pseudomonal sepsis is a more rare occurrence $[3,4]$.

The initial cutaneous findings of purpura fulminans are painful areas of erythema that are often reversible with treatment of the underlying infection. As the condition progresses, the areas become characteristically stellate in shape as a result of intravascular thromboembolic events. Lesions may become bullous, and eventually necrotic. Once full thickness necrosis has developed, patients require more extensive treatment, longer recovery time, and have poorer outcomes, including amputation and death [1]. Thus, purpura fulminans is a hematological and dermatologic emergency that requires urgent diagnosis and intervention.

Diagnosis is primarily clinical, based on typical skin lesion appearance as well as laboratory findings (elevated D-dimer, decreased platelets, etc.) [1]. Skin biopsy can be performed to support the diagnosis, and would include the presence of thrombi in dermal vessels, extensive intravascular platelet aggregates, and massive vascular congestion [2]. However, empiric treatment should be started without delay. 
Proper treatment for purpura fulminans in the context of sepsis includes antibiotics and surgical management to prevent further tissue death and decrease the likelihood of future amputations [5]. Both were utilized in the care of the described patient. Treatment targeting the coagulation cascade abnormalities found in purpura fulminans is also considered standard, including platelet transfusion for severe thrombocytopenia (platelet count $<50 \times 10 \% \mathrm{dl}$ ) and replacement of coagulation factors [1]. The combination of thrombomodulin (a cofactor for thrombin) and fresh frozen plasma has also been found to be effective in contributing to the resolution of purpura fulminans [6]. Isolated protein C supplementation is a newer method used to successfully restore anticoagulant factors consumed in the disease process [7]. Despite the above array of options for reversing coagulation pathway abnormalities, and their shown benefits in purpura fulminans, none were used in the treatment of our patient. Yet, the patient survived and was discharged from the hospital.

\section{CONSENT}

The examination of the patient was conducted according to the Declaration of Helsinki principles.

\section{REFERENCES}

1. Chalmers E, Cooper P, Forman K, Grimley C, Khair K, Minfor A, et al. Purpura Fulminans: recognition, diagnosis, and management. Arch Dis Child. 2011;96:1066-71.

2. Lerolle N, Carlotti A, Melican K, Aubey F, Pierrot M, Diehl J, et al. Assessment of the Interplay between Blood and Skin Vascular Abnormalities in Adult Purpura Fulminans. Am J Respir Crit Care Med. 2013;188:684-92.

3. Khalid S, Sill J, Mahmood J. The Secret Affair Between Pseudomonas and Purpura Fulminans. Am J Respir Crit Care Med. 2017;195:A1858.

4. Harikrishna J, Mohan A. Infectious Purpura Fulminans. Indian J Med Res. 2015;141:130-1.

5. Childers B, Cobanov B. Acute Infectious Purpura Fulminans: A 15-Year Retrospective Review of 28 Consecutive Cases. Am Surg. 2003;69:86-90.

6. Hayami T, Yamaguchi A, Kato T, Tanaka T, Nishizawa Y, Yanagi T, et al. Purpura Fulminans in congenital protein $\mathrm{C}$ deficiency successfully treated with fresh frozen plasma and thrombomodulin. J Dermatol. J Dermatol. 2017 Dec 19. doi: 10.1111/13468138.14194 .

7. Schellongowski P, Bauer E, Holzinger U, Staudinger T, Frass M, Laczika K, et al. Treatment of adult patients with sepsis-induced coagulopathy and purpura fulminans using a plasma-derived protein C concentrate (Ceprotin). Vox Sang. 2006;90:294-301.

Copyright by Leah Ellis Wells, et al. This is an open-access article distributed under the terms of the Creative Commons Attribution License, which permits unrestricted use, distribution, and reproduction in any medium, provided the original author and source are credited.

Source of Support: Nil, Conflict of Interest: None declared. 